\title{
ECONOMIC INFORMATION SYSTEMS INTEROPERABILITY
}

\author{
Vasile Irimia $^{1}$
}

\begin{abstract}
In this article we define and describe the economic information interoperability problem and how it affects today's enterprises in the context of globalization and current ICT development, the currently used solutions found in the integration and interoperability of information systems literature (EDI, Web Services, ebXML, RosettaNet, XBRL), the main research activities done so far in the field of Enterprise Interoperability and the observed trends in the evolution of standard solutions.
\end{abstract}

Keywords: interoperability problem; economic information system; standard; enterprise interoperability

JEL codes: D83, D85, M15, O14, O31, O33

\section{Introduction}

This article serves as an introduction to economic information systems interoperability field, describes the current problems of interoperability faced by the enterprises, the state of the art in the solutions landscape (Web Services, ebXML, RosettaNet, XBRL), the main research activities done so far and observed trends as they result from researching the current solutions.

The methodology used to compile this information was a bibliographic review of the Enterprise Interoperability research field having as primary input the economic information system theory, the European Union research agenda (Enterprise Interoperability Research Roadmap, $7^{\text {th }}$ Framework Programme) and relevant research projects (e.g. Abilities project), the literature available on information systems integration and also from a doctoral study on current enterprise interoperability standards conducted as part of my $\mathrm{PhD}$ program and published as my first $\mathrm{PhD}$ student report (Irimia V., 2011).

Among the most relevant related work on this topic we mention the Enterprise Interoperability Research Roadmap, versions 4 (Man-Sze Li et al, 2006) and 5 (Yannis Charalabidis, et al., 2008), which have served as input for the European Framework Programme 6 and the current FP7. In FP6, Enterprise Interoperability was part of the "ICT for creating networks of enterprises". The Networked European Software and Services Initiative (NESSI) has developed a strategic research agenda which identifies interoperability as a generic aspect of its technological domains. Another important work is the Athena Framework. Also, Johan Ullberg discusses the barriers to enterprise interoperability (Johan Ullberg et al, 2009).

In this article we aim to present an integrated view with information from a current literature and field review on enterprise information system interoperability and integration, describe the interoperability problem, the solutions found in the literature and the trends observed.

This article is structured as follows: first part introduces a few needed concepts; the second part introduces the enterprise interoperability problem; the third part presents to state of the art solutions present in the literature on information system integration and in interoperability; the fourth part presents the researched already done as stated in the Enterprise Interoperability field research plan; some trends from the analysis of current standards used to enable interoperability; and we end with conclusion.

\footnotetext{
1 “Alexandru Ioan Cuza” University of Iaşi, Doctoral School of Economics, Romania, e-mail: irimia.vasile@gmail.com
} 


\section{Concepts}

Enterprise Interoperability is a relatively new term for a new domain of activity that aims to improve the way in which enterprises, with the help of Information and Communication Technology, interoperate with other enterprises, organizations or other business units within the same enterprise, to achieve its goals.

The Interoperability is defined by the IEEE as the common execution of a task by two or more different systems.

D. Oprea defines the economic information system as "an assembly of human resources and capital, invested in a economic unit, with the purpose of collecting and processing the data needed to produce information that will be used at all decision levels of management and control of the organizations activities" (D. Oprea, 2005).

Economic information system interoperability can be seen as the easy connection and communication between two different enterprise information systems, within a network of suppliers and business partners, with the help of the new information technologies.

\section{The enterprise interoperability problem}

According to the Enterprise Interoperability Research Roadmap (Man-Sze Li et al., 2006), enterprise interoperability has become a strategic requirement in all industries. The future enterprises will be characterized by the ability to collaborate, adapt and interoperate. The strategic i2010 Framework has identified explicitly interoperability as a key bottleneck that must be tackled. This framework is the logical link between the high level goals of the Lisbon Strategy and more operational activities related to ICT. The Lisbon Strategy emphasizes the importance of market efficiency to achieve high productivity which is a key requirement for economic growth and social welfare. The i2010 framework acknowledges that enterprises achieve productivity gains with the help of ICT but they face a lack of interoperability, security and trust, having difficulties integrating ICT at the workplace and high cost. Interoperability is identified explicitly as one of the bottlenecks that must be removed by the i2010 Framework to make European Union more competitive.

The electronic business is one of the domain closest related to the requirements of interoperability. Stephen Mohr apud Buchman (Buchman, 2007), affirms that the electronic business is the fundamental exercise in interoperability of whose success depends the large scale development of collaborative systems. Although more than $90 \%$ of the enterprises are connected to the Internet, studies show that in 2005, only $33.5 \%$ use electronic business solutions to automate internal processes and only $15 \%$ use them to integrate with suppliers and clients. Only $10 \%$ of the enterprises use them to integrate with their suppliers and $9 \%$ use them to integrate with clients (Man-Sze Li et al, 2006). According to Europe's Digital Competitiveness Report in 2010, 34\% of EU enterprises use ICT to exchange information with business partners (twice as much since 2005) and $15 \%$ used ICT for supply chain management. Although they would most benefit, small and medium sized enterprises (SMEs) face even more challenges in adopting interoperability solutions, the gap between them and the large enterprises being significant. Only $30 \%$ of the enterprises that use electronic business solutions are SMEs. The conclusion is that the usage of IT in business is still far from being a routine for the European enterprises and although most of the European enterprises are SMEs, the usage percent here is smaller by a factor of 3 and more. Large companies adapt much slower to the market changes. Small and medium sized enterprises, although they are very flexible, they do not have the required resources to monitor the market changes. SMEs do not have the same financial possibilities as the large ones, limiting the potential for interoperability if that is even considered. The SMEs face significant barriers entering new markets and collaboration networks. The time, effort and costs of interoperability are disproportionate with the size and operation area.

A main problem of interoperability is that it was not based on scientific grounds but has evolved bottom-up, ad-hoc, using proprietary and local solutions. Creating links between enterprises that use this kind of solutions, using middleware translators, is particularly costly and 
time consuming. The time and money needed to integrate with a partner may exceed the opportunity potential. Alignment between business and technical level is largely missing. Managers have difficulties in taking decisions regarding enterprise interoperability. This is in part due to the traditional way of doing business: business based on personal relations, transactions handled face to face and decision taken with human intervention.

The current enterprise interoperability problem has to do with the adaptation to globalization, faster change and innovation management. Buchman affirms that the problems of interoperability are caused by: legacy systems, technical heterogeneity and divergent standards (Buchman, 2007). Also, the investments in legacy systems are still high so replacing them is not an option right now (Buchman, 2007). The integration applications that were previously solution are now a barrier for collaboration between enterprises.

Enterprise Interoperability can increase the SMEs ability to enter new markets by reducing the barriers to collaborate, increasing the enterprise ability to exploit new business opportunities and increasing the enterprise flexibility. Enterprises will have increase access to information, knowledge, technologies and skills. This will lead to increased performance, more and better products and services, lower costs and time to market, increased speed.

The Enterprise Interoperability Framework (EIF) defines three types of interoperability (Johan Ullberg, 2009): conceptual (syntactic and semantic), technical and organizational. These types affect the following levels of interoperability: data, services, processes, and business. By combining these, Johan Ullberg obtains the barriers to interoperability: different semantics of data (data semantics), different syntax of data (data syntax), different data formats (data - technical), who owns the data (data - organizational), different services (service - content, service - syntax, service - semantics), different processes (process - content, process - syntax, process - semantics, process - technical, process - organizational), different businesses (business - content, business syntax, business - semantics, business - technical, business - organizational).

Interoperability is an essential requirement for information communication. At a first glance we can distinguish between a conceptual level and the technical level. At the conceptual level, the collaboration is achieved by sending messages within a predefined business process. At the technical level, we see the hardware and software components and their communication interface. Johan Ullberg et al. distinguishes one more level here, the organizational level, but we consider this as a part of the conceptual level (Johan Ullberg et al., 2009). Buchman distinguishes a formatting level, a structure level and a high level (semantic) (Buchman, 2007). We believe he refers to the various levels of language (symbols, messages, semantics), covering more the conceptual level than the technical.

\section{Solutions}

Today, there a many technological solutions that claim to solve the enterprise interoperability problems. The main problem of these solutions is that they are based on proprietary protocols and formats. This leads to islands of interoperability and captive markets. It is recognized that these are not appropriate in a global dynamic market and they are inefficient in real business. Problems of enterprise interoperability have occurred and have been discussed for over a decade in a large number of communities, interoperability still remaining a problem for enterprises. The integration projects are complex and expensive and there is still a gap between the technical and business levels. In the last decade there has been significant progress in the field of enterprise interoperability. We have witnessed the emergence of many frameworks and standard specifications like: Web EDI, Open EDI, ebXML, RosettaNet, OAG integration specification, FIPA architecture and its associated specification for creating software agents, Service Oriented Architectures and their Web Services, Semantic Web Services, languages for enterprise and business process modeling, Biztalk, XBRL (Man Sze-Li et al., 2006). The current technological developments and standardization activities related to enterprise interoperability are mostly fragmented. There are 
many standards and standard producing organizations (OASIS, OMG, UN/CEFACT, CEN, RosettaNet, GS1, etc.). Current implementations are fragmented, have a too narrow applicability and lack the critical mass. Recent studies show that the ICT industry is moving away from proprietary solutions and towards web based standards. (Man Sze-Li et al., 2006)

It is recognized that open-source development is a key element for interoperability, having a positive impact on adoption of the solutions based on reference standards (Man Sze-Li et al., 2006).

The EDI standards have been the first attempt to enable electronic business by language interoperability and are, currently, despite many rumors, still dominant. According to Microsoft, it represents more than $75 \%$ percent of all electronic B2B transactions (Microsoft, 2011). Unfortunately, one of its main problems is just this: there is more than one standard: there are many EDI standards. Every industry sector has organizations that created specific standards to enable business-to-business transactions. Examples are: ODETTE in the auto industry, CIDX in the chemical industry, GS1/UCC in retail, EURATEX in textile, HL7 in health, IAI in constructions, OTA in traveling, PIDX in petroleum industry, etc.

ebXML is one of the most recent public standards for electronic commerce, developed through a joint effort between UN/CEFACT and OASIS using an open process, where public contributions are encouraged and welcomed. It is inspired by EDI standards and supported by XML and the new technologies brought forth by the Internet. Its vision is to create a global electronic market where enterprises of all sizes from all around the globe can meet and conduct business. This vision has inspired a powerful solution. ebXML aims to be a complete solution for electronic commerce. As such, it develops specifications for the entire infrastructure needed to support electronic commerce at a global scale. It aims to ease the integration effort and fosters dynamic automatic business relationships using a standard platform.

RosettaNet is an organization of standardization, member of GS1 US, which promotes electronic commerce through the specifications known as the RosettaNet standards. RosettaNet recognizes the need for interoperability in the supply chains and wants to promote the wide spread of standard business processes. For this purpose it develops universal open standard specifications for the supply chains. The development of the standards is made through an open process driven by industry initiative to solve its own needs. RosettaNet strives to enable enterprises of all sizes to collaborate by electronic means, conduct business and share information in an extended supply chain. The RosettaNet standards are focused on the high-tech semiconductor industry.

Web services have become very popular, enjoying support from all major software vendors like Microsoft, Oracle, IBM, HP, etc. The main contribution of web service standard is creating a messaging protocol independent of a particular implementation. Unfortunately, to truly be a business messaging solution, we need a higher level standard that addresses the problems not addressed by the Web Service specification, like ebMS from ebXML or RosettaNet transmission component. The web service standard is still too young as the WS-I Basic Profile specifications and the myriad of WS-* related standards prove it. SOA is not a standard specification but an application architecture style based on loose principles. As it currently stands, it is far from being an interoperability solution.

Business financial reporting has been an important topic for standardization. This is due to the need for cross-boundary data analysis and integration that enable economic decisions. There are two major standardization efforts in this area: one is the International Financial Reporting Standards - IFRS and the other one is its complement - XBRL, the eXtensible Business Reporting Language.

\section{Research so far}

Research efforts so far have focused on frameworks and technical aspects of interoperability like: architectures and frameworks for systems interoperability, Service Oriented Architectures and Enterprise Interoperability, design and execution of interoperable services, semantic services and frameworks based on ontologies, methods and instruments for collaboration, approaches and 
solutions for model driven interoperability, approaches and instruments for modeling Enterprise Applications Interoperability, business and industrial strategies for Enterprise Interoperability, interoperability in product design and product engineering, and others. Architectures and frameworks for interoperability have been analyzed and new approaches have been promoted based on characteristics like: peer-to-peer interconnection, data integration, service integration, processes alignment, operation efficiency, start cost, and others. The legal framework problem and the integration of legal rules into the design and implementation of interoperability solutions have been analyzed. Technologies like SOA, MDI, BPMN, BPEL, CCTS and UBL have been tested revealing even more the need for standardization in domains like: inter-organizational business processes, document exchange, service integration and web service orchestration. (Yannis Charalabidis et al., 2008)

Some projects like INTEROP-VLAB were aimed at stimulating the adoption of interoperability in the market. IVLAB is a reference in the Enterprise Interoperability field and is consulted by international organizations like IMS and IFIP. The Athena project goal was to create the Centre for Enterprise Interoperability which is a platform for the definition and application of the integration methodologies and instruments, supporting existing standards where possible and promoting the creation of public standard business processes to support cross-industry interoperability. (Yannis Charalabidis et al., 2008).

Projects like FP7 COIN IP have researched new business models and scenarios, some based on principles and design templates of Web 2.0 like software-as-a-service combined with the concept of interoperability as a utility service. (Yannis Charalabidis et al., 2008).

There is a need to move away from the architectures and software solutions focused on the enterprise towards solutions and architectures of loosely coupled networks of enterprises that are better able to adapt to the markets changes. The majority of the technical problems regarding the interoperability have been solved. The main problem remains what and how the business partners communicate.

Current research projects aim to make it easier and cheaper for the SMEs to interoperate with other enterprises to transform faster new ideas into products and services that generate income, increasing their global competitiveness. It is hoped that the size of the market will count less than the ability of the enterprise to collaborate, adapt and interoperate.

\section{Trends}

The main trends in the development of standard solutions for economic information systems interoperability are: using XML and XML derived languages; the reuse of existing standards in new evolved standards; a move towards standards that solve real economic problems and away from technical standards; focus on creating languages; focus on standardization of processes; harmonization and convergence between standards; increased cooperation between standard producing organization; the need to create a universal or unified language; developing of solutions for gradual implementation of complex interoperability solutions (Irimia V., 2011).

\section{Conclusion}

Simply put, interoperability is the ability of an enterprise to easily collaborate with other enterprises or internal organizational units using the new information technologies. Today, interoperability is considered a key bottleneck to the success of European enterprises in the current context of globalization that must be addressed. It is believed that enabling interoperability can produce outstanding results in terms of economic growth. The current enterprise interoperability problem has to do with the adaptation to globalization, faster change and innovation management. According to European Union reports, ICT usage in enterprises is still far from being a routine for the European enterprises. Enterprises achieve productivity gains with the help of ICT but they face a lack of interoperability. Today there are many solutions that claim to solve the interoperability 
problem: EDI standards, ebXML, RosettaNet, Web Services, GS1 standards, XBRL, OAG integration specification, FIPA architecture and its associated specification for creating software agents, Biztalk, etc. Even so, interoperability still remains a problem. Solutions have been tested revealing even more the need for standardization in domains like: inter-organizational business processes, document exchange, service integration and web service orchestration. Today there are many research and development initiatives aimed at solving the various problems of enterprise interoperability. My current research of the enterprise interoperability field of research and development and the current standards and solutions available shows clearly that although there are many more research and development initiatives related to interoperability, the main problem faced by the enterprises has not changed significantly and we are only in an early phase of solving it . So what we have now is a better understanding of the interoperability problem, the solutions and the relation between what the solutions solve and what they don't, both in theory and in the market (Irimia V., 2011). Current research projects aim to make it easier and cheaper for the SMEs to interoperate with other enterprises. In the evolutions of standard solutions we observe a trend towards XML and XML derived languages, promotion of open standards, the reuse of existing standards in new evolved standards; a move towards standards that solve real economic problems and away from technical standards; focus on creating languages; focus on standardization of processes; harmonization and convergence between standards; increased cooperation between standard producing organization; the need to create a universal or unified language; developing of solutions for gradual implementation of complex interoperability solutions.

\section{Acknowledgment}

This work has been done with the financial support from PODRU/88/1.5/47646 project, cofinanced from European Social Fund through the Sectorial Operational Program: Developing of Human Resources 2007-2013.

\section{References}

1. Buchman R. A., 2007. Rolul XML in interoperabiltatea sistemelor informatice pentru afaceri, Cluj-Napoca, Risoprint.

2. Charalabidis Y., Gionis G., Hermann K. M., Martinez C., editors, 2008, Enterprise Interoperability Research Roadmap, version ftp://ftp.cordis.europa.eu/pub/fp7/ict/docs/enet/ei-research-roadmap-v5-final_en.pdf, accessed on 31.03.2011

3. Irimia V., 2011. Standarde de Interoperabilitate, phd report, unpublished

4. Man-Sze Li, Cabral R., Doumeingts G., Popplewell K., editors, 2006, Information Society Technologies, Enterprise Interoperability Research Roadmap Final Version, pp 4,

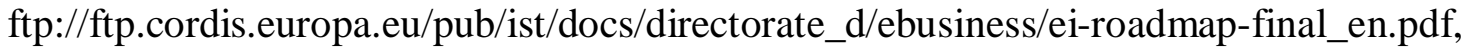
accessed on 31.03.2011

5. Oprea D., Mesnita G., Dumitriu F., 2005. Analysis of Information Systems, Editura Universitatii Al.I.Cuza, Romania, Iasi

6. Ullberg J., Chen D., Johnson P., 2009. Barriers to Enterprise Interoperability, Lecture Notes in Business Information Processing, 1, Volume 38, Enterprise Interoperability, Part 1, Pages 13-24

7. ATHENA Framework, http://modelbased.net/aif/, accessed on 31.03.2011

8. Europe's Digital Competitiveness Report 2010. http://ec.europa.eu/information_society/ digital309agenda/documents/edcr.pdf - accessed on 31.03.2011

9. Microsoft, 2011. B2B Integration, http://www.microsoft.com/biztalk/en/us/b2bintegration.aspx - accessed on 3.01.211 